\title{
Scintigraphic Diagnosis of Infectious Complications in Renal Failure Patients Undergoing Hemodialysis, Continuous Ambulatory Peritoneal Dialysis or Renal Transplant
}

\author{
Ana María García Vicente*, Sebastián Ruiz Solís, Angel Soriano Castrejón, Víctor Manuel \\ Poblete García, Maria del Prado Talavera Rubio, Sonia Rodado Marina, Montserrat \\ Cortés Romera. \\ Department of Nuclear Medicine; Ciudad Real General Hospital; C/ Tomelloso s/n; Ciudad Real - Spain
}

\begin{abstract}
Patients with end-stage renal disease have two therapeutic options, dialysis and renal transplantation. Infectious complications occurring in such patients will not only condition the effectiveness of such treatments, but are among the main causes of morbidity and mortality in such cases. Knowledge of the advantages and limitations of nuclear techniques is essential for management of these conditions.
\end{abstract}

Key words: Infectious complications, hemodialysis, peritoneal dialysis, renal transplantation, ${ }^{67}$ Gallium, labeled leukocytes

\section{INTRODUCTION}

Patients suffering chronic end-stage renal disease, approximately four out of every 10,000 people, have different therapeutic options, each having its own advantages and disadvantages, without which they would not be able to survive. Of the two types of dialysis, the most widely used is hemodialysis (HD), in up to $90 \%$ of cases. While there are no significant differences between HD and peritoneal dialysis as regards results, their complications, particularly infections, are substantially different and greatly significant, as they may result in failure of one or the other procedure. Infections associated with the venous or peritoneal catheter are the most important complications and the leading cause of morbidity in these patients.
Renal transplantation (RT) is the procedure of choice for these patients, and the most common transplant surgery today. Annual mortality from infection has currently decreased to less than 5\% (Simon et al., 2001). However, infection is still a serious problem because of a dual impact on RT, direct morbidity and mortality from infection and the indirect effects this exerts on rejection mechanisms that may promote development of acute rejection or activation of a latent cytomegalovirus (CMV) infection. Few studies have assessed infectious complications using radioisotopic techniques. Hence, an in-depth review of the true value of Nuclear Medicine in such conditions is presented.

\footnotetext{
${ }^{*}$ Author for correspondence
} 


\section{INFECTIOUS COMPLICATIONS IN HEMODIALYSIS}

Infectious complications of the vascular access are the most common cause of morbidity and mortality among HD patients. They account for $48 \%$, and in some series for up to $73 \%$, of all bacteremias (Kessker et al., 1993; Marr et al., 1998). It has, therefore, been estimated that infectious complications may be responsible for up to $36 \%$ of the deaths in these patients (Mailloux et al., 1991). In addition, most of these bacteremias are caused by Staphylococcus, and are, therefore, associated with high rates of mortality, recurrence, and severe metastatic complications (Hoen et al., 1998; Kairaitis et al., 1999). The most common complications include infectious endocarditis, septic arthritis, epidural abscess, septic pulmonary embolism, and osteomyelitis (Robinson et al., 1997; Obrador et al., 1996; Kovalik et al., 1996). The incidence of infections related to venous access increases in central venous catheters, while native arteriovenous fistulas have a lower risk of infection (Powe et al., 1999; Kaplowitz et al., 1998).

Therefore, when faced with a patient on HD with fever, even in the absence of local clinical signs of infection, an infectious complication of the vascular access should be suspected. Such infections may be of two types.

\section{Infections related to the catheter exit site}

These are common complications affecting $8 \%$ to $21 \%$ of the patients having a venous access, and are a significant cause of access loss (Moss et al., 1990). In catheters with a tract or tunnel, they are classified as exit site infections and tunnel infections. The former are infections external to the cuff, while the latter extend through the tunnel proximal to the cuff and are the most severe, because of their frequent association to bacteremia (Schwab et al., 1999).

Catheter-associated bacteremia may cause various clinical signs and have an acute or torpid onset, particularly in elderly or immunosuppressed patients. Exit hole infections usually cause local symptoms (redness, exudate, scab) and may be treated conservatively if they are not associated with tunnel infections or bacteremia. By contrast, tunnel infections require catheter removal and implantation of another venous access at a new site (Beathard, 1999).

\section{Infections of arteriovenous fistula}

Arteriovenous access consists of the native arteriovenous fistula and the synthetic arteriovenous graft (Figs. 1 and 2). These infections are usually clinically recognized because of the local signs they induce. However, the absence of such signs and symptoms does not rule out the possibility of a clinically silent infection, particularly in cases of unexplained sepsis, leukocytosis, or fever of an unknown origin (Nassar et al., 2001).

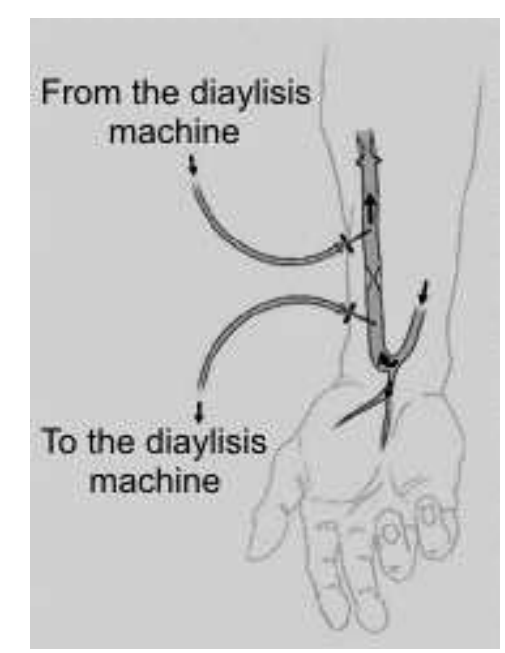

Figure 1 - Diagram of a native arteriovenous fistula.

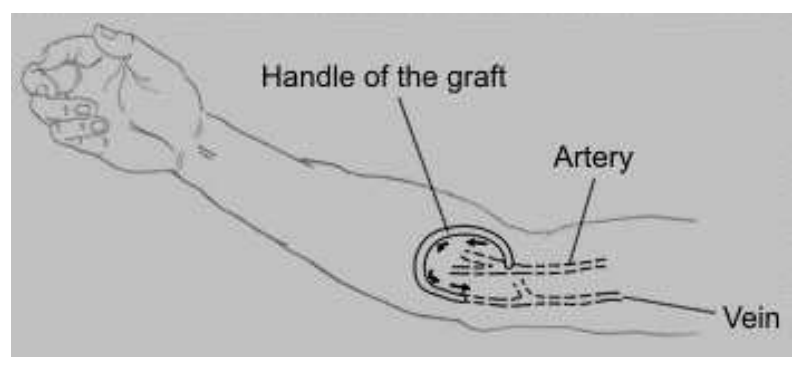

Figure 2 - Diagram of a synthetic arteriovenous graft.

Polytetrafluoroethylene grafts in HD patients are not usually removed when they are no longer used and are thrombosed, so that some patients may have one or several grafts. These grafts, initially considered safe, have been recognized as potential reservoirs of occult bacterial infection that may result in serious infectious complications (Ayus et al., 1998). Such infections of thrombosed or clotted arteriovenous grafts tend to be silent and 
are therefore difficult to diagnose, unless suspected (Nassar et al., 2000).

\section{Scintigraphic diagnosis of infectious complications in HD}

While scintigraphy with ${ }^{99 \mathrm{~m}} \mathrm{Tc}-\mathrm{HMPAO}$-labeled leukocytes is currently considered the nuclear medicine procedure of choice for diagnosing vascular prosthesis infections, no studies using this radioactive drug to assess infectious complications in patients on HD (Prats et al., 2005) were encountered. Studies found reporting the use of ${ }^{111}$ Indium for leukocyte labeling define this procedure as a very useful tool for investigating the possibility of graft infection. A diagnostic precision close to $100 \%$ has been reported even when there are no local signs of infection (Lawrence et al., 1985; Palestro et al., 1990, Meller et al., 2002).

Ayus et al. (1998) evaluated the prevalence and clinical relevance of infections of clotted arteriovenous grafts. For this purpose, they studied 41 patients with such grafts. Twenty patients had fever with/without clinical signs of associated sepsis (group 1), while the rest (21 patients) were asymptomatic and were considered as the control group (group 2). All patients underwent scintigraphy with ${ }^{111}$ Indium-labeled leukocytes and subsequent surgical removal of the graft. Bacterial cultures were performed of both the graft and blood samples. All group 1 patients and 15 patients from group 2 showed positive uptake in or around the graft area. The significance of such findings was demonstrated when purulent material was recovered from all group 1 patients and from 13 of the 15 patients from group 2 with a positive scintigraphy. Thus, these authors obtained a $100 \%$ sensitivity and a $75 \%$ specificity.

\section{INFECTIOUS ASSOCIATED COMPLICATIONS AMBULATORY PERITONEAL DIALYSIS (CAPD)}

Peritoneal access using a special catheter is the key element for success of this dialysis procedure, and is at least as important as vascular access in hemodialysis. This catheter is a foreign body, and may be a source of infection by behaving as a bacterial reservoir in both the catheter exit hole and subcutaneous tunnel (Fig. 3).

In addition, passage of the causal microorganism into the peritoneal cavity through the catheter exit hole and tunnel is still considered the main entry portal of infection in cases of peritonitis associated to CAPD (Piraino et al., 1997). There are two types of infections, those affecting tissues close to the catheter, and those involving distant sites as a result of pathogen dissemination.

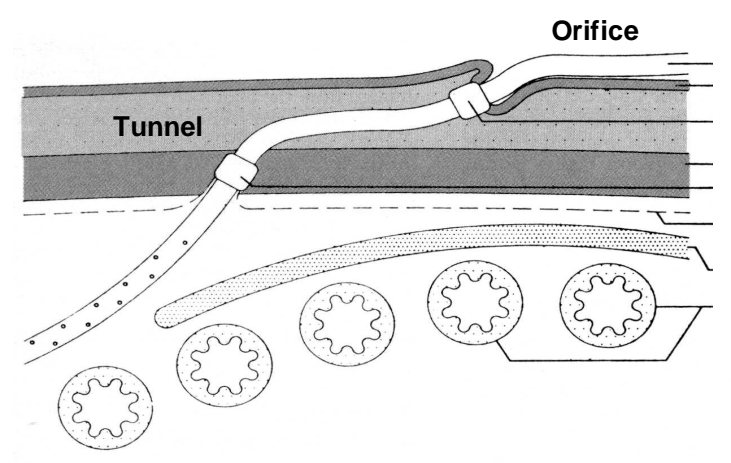

Figure 3 - Diagram of CAPD catheter implantation.

\section{Infection of the catheter exit hole and/or tunnel}

The chance of suffering an infection of the catheter exit hole has been estimated to be $46 \%$ in the first year, and $70 \%$ three years after initiating CAPD. Moreover, it is not uncommon for this event to result in peritonitis (30\%-50\%) and catheter removal (15\%-57\%) (Vargemezis et al., 2001).

A normal exit hole shows a normal skin appearance, with no inflammatory signs. A slight serous discharge may exist. An infected hole appears reddened, with suppuration, inflammation, and formation of granulation 
tissue. In such cases, diagnosis is based on bacterial growth in the culture of discharge from the hole and is supported by the presence of polymorphonuclears in a Gram stain (Montenegro et al., 1997). However, the presence of positive cultures in the exit hole, in the absence of inflammation, does not suggest infection (Twardowski, 1993).

Catheter tunnel infection may occur as an extension of infection of the exit hole with pain, swelling, nodularity, and redness on the subcutaneous portion of the catheter, and even systemic symptoms such as fever. The microorganism causing tunnel infection may induce relapsing peritonitis. The most common causative microorganisms include: Staphylococcus aureus (25\%-85\%), mixed flora including $S$. aureus (16\%-35\%), Gram-negative enteric germs $(7 \%-11 \%)$, and fungi $(1 \%-3 \%)$ (Keogh et al., 1998).

\section{Peritonitis}

This complication occurs in 15\%-35\% of hospital admissions of these patients and is the most common reason for switching to hemodialysis $(40 \%-45 \%)$. It is also associated with a patient death rate of $7 \%-10 \%$ (Vargemezis et al., 2001).

Clinical diagnosis is based on the triad consisting of abdominal pain and signs of peritoneal irritation, peritoneal fluid turbidity (>100 cells $/ \mathrm{ml}$, with $>50 \%$ neutrophils), and a positive culture of peritoneal fluid. A diagnosis of peritonitis may be made when two of these three signs or symptoms are found.

Peritonitis is relatively easy to diagnose in these patients based on the evidence provided by both the clinical signs and symptoms, and the culture of peritoneal fluid. However, infections of the catheter exit hole and tunnel are more difficult to diagnose because of the possibility of obtaining positive cultures in the absence of infection, as well as the paucity of clinical signs and the difficulty to adequately assess the external appearance of the exit hole in certain cases.

\section{Scintigraphic diagnosis of infectious complications in CAPD}

Few studies with labeled leucocytes allowing for an evaluation of their diagnostic efficacy in catheter-associated infectious complications have been documented in patients undergoing CAPD. All studies published to date have used
${ }^{111}$ Indium-labeled leukocytes, which have been shown to be useful for differential diagnosis between infection of the catheter tunnel and infection of the exit hole (Kipper et al., 1984). This is a clinically important differentiation, because the catheter should be removed in tunnel infections.

Becker et al., (1987) showed that ${ }^{111}$ Indiumlabeled leukocytes had a $91 \%$ precision for diagnosing intraabdominal abscesses and renal cyst infections in patients on peritoneal dialysis. In the latter, this procedure is very superior to other diagnostic modalities, such as ultrasonography and MRI, because of its greater specificity.

A study (Ruiz et al., 2004) was recently conducted by this group to assess the value of abdominal scintigraphy with ${ }^{99 \mathrm{~m}} \mathrm{Tc}-\mathrm{HMPAO}-$ labeled leukocytes in the diagnostic algorithm for infectious complications associated with CAPD. A total of 35 studies were performed on 17 patients on CAPD, which were divided into three groups: a control group (5), a group of studies in patients with clinically suspected infection of the catheter exit hole and/or tunnel (18), and a group with clinically suspected peritonitis (12). An abdominal scintigraphy with ${ }^{99 \mathrm{~m}} \mathrm{Tc}-\mathrm{HMPAO}-\mathrm{labeled}$ leukocytes was performed on all patients. The results were compared with the final clinical diagnosis, for which an evaluation was made of the external appearance of the exit hole or dialysis fluid, a culture of material or discharge from the exit hole and dialysis fluid, and the clinical course. In the studied sample, sensitivity and specificity values for diagnosis of exit hole infection of $83 \%$ and $75 \%$, respectively, were obtained, as well as a $100 \%$ sensitivity for diagnosis of peritonitis. A case of catheter tunnel infection was confirmed by culture, and no pathological uptake in any of the control group cases was observed.

\section{INFECTIOUS COMPLICATIONS IN RENAL TRANSPLANT}

It is estimated that more than $75 \%$ of transplanted patients experience some type of infection in the first year after a RT (Rubin et al., 1988). The risk of infection by specific pathogens is greater in certain periods and follows a predictable pattern, dictated by immunosuppression type and intensity. Rubin et al. (1988) described three periods. In the first 
period, during the first month after RT, infections are related to complications derived from surgery and are often acquired at the hospital, with a predominance of urinary tract infections (UTIs) and surgical wound infections. The second period ranges up to the sixth month. Opportunistic infections by viruses and fungi predominate in this period as a result of the intensity of immunosuppressant therapy, causing an impaired cell-mediated immunity. The third period extends from the sixth month onwards, a time when immunosuppressant therapy is less aggressive and infections would be the same as in the general population. The most common infectious complication in RT is UTI, followed by other infections involving lung (9\%), surgical wound $(5 \%)$, oral cavity (2\%), central nervous system (1\%), and esophagus (1\%) (Wyner et al., 1994; Franz et al., 1992).

\section{UTI}

The prevalence of UTI after hospital discharge following a RT ranges from $28 \%$ to $90 \%$, which is much higher than in the general population, where prevalence is approximately 20\% (Wyner et al., 1994; Burgos et al., 1999). UTI incidence in the first six months following RT has decreased from $30 \%-60 \%$ to less than 5\%-10\% with the use of antibiotic prophylaxis (Simon et al., 2001; Fishman et al., 1998), although some authors have found higher rates in the first month (70\%). As a result, UTI's may cause up to one third of bacteremias (Abbott et al., 2001). Factors contributing to an increased infection risk include use of urinary catheters, urethral stents, HD prior to RT, diabetes, polycystic kidney, and vesicoureteral reflux (Schmaldienst et al., 2002). Women have a twofold risk compared to men. In addition, UTI relapse or persistence is significant, since half the patients with a first UTI episode following RT will experience at least a second episode (Ramsey et al., 1979).

Late infections after RT, considered as "benign" some years ago, are not so benign (Muller et al., 1998). A retrospective study on 28,942 US patients found that late UTIs were associated with an increased mortality risk (Abbott et al., 2004).

Clinically, UTI's occur as graft pyelonephritis, bacteremia, persistent infections, or frequent reinfections. Since infection is often asymptomatic and no pyuria is found in the sediment, a high degree of suspicion and regular urine cultures are required for diagnosis (Franz et al., 1992). By contrast, fever of an immunological origin as a result of RT rejection should be suspected as the first possibility in patients with a febrile syndrome who have discontinued or decreased immunosuppressive therapy (Torregrosa et al., 1994).

Patients receiving RT with an underlying polycystic disease often have severe infection of the native and even the transplanted kidney. Immunosuppressive therapy may mask signs of inflammation and make infection location difficult, which may result in a systemic infection (Tsang et al., 1989). Moreover, ultrasonography and CT are not capable of differentiating infected from non-infected cysts.

\section{Scintigraphic diagnosis of infectious complications in RT}

Scintigraphy with ${ }^{67}$ Gallium has been shown to be of value for assessing abdominal inflammatory lesions with a diagnostic precision ranging from $86 \%-90 \%$ in the diagnosis of renal infections (Hurwitz et al., 1976). At approximately 24 hours, $10 \%$ of the administered dose remains in the plasma. Kidneys excrete $10 \%-25 \%$ of the dose, mainly during the first 24 hours, after which the liver and gastrointestinal tract are the main excretion routes (Park et al., 1977).

While this excretion does not always consistently occur, renal activity may be intense during the first 24 hours, but is usually mild or absent at 48-72 hours after injection (Neumann et al., 1996). García et al. (1984) observed that $50 \%$ of the patients without nephropathy had a mild kidney uptake (lower than liver uptake).

The capacity of ${ }^{67}$ Gallium to accumulate in active inflammation areas has been shown to be of value in diagnosis of renal inflammation (Wood et al., 1978; Linton et al., 1980; Kumar et al., 1976; Mendez et al., 1980; Yen et al., 1999), particularly in acute conditions (Lin et al., 2000). The exact mechanism of binding in inflammation sites is not well defined. Based on experimental evidence, its deposit is related to different mechanisms: increased vascularization, protein accumulation, labeled leukocytes, and microorganisms. Adequate antibiotic therapy leads to a decreased leukocyte migration simultaneously resulting in a decreased ${ }^{67}$ Gallium uptake. However, while leukocyte 
infiltration starts at 30 minutes and peaks at 24 hours, ${ }^{67}$ Gallium uptake increases with the evolution time of the infectious site up to a maximum period of 5 to 15 days. These observations suggest that ${ }^{67}$ Gallium accumulation may be a more complex event in which different mechanisms are involved (Tsan et al., 1978; Gelrud et al., 1974).

Under inflammatory conditions, an adequate accumulation of ${ }^{67}$ Gallium in the kidney within 2-6 hours after injection or an increased uptake in sequential images occurs (Park et al., 1977). Pyelonephritis usually occurs as a diffuse accumulation involving the entire kidney, but focal accumulations may exist, although this is less common (Brugh et al., 1979). Demonstration of an inflammation area in the perinephritic space or early uptake in a mass is more suggestive of an abscess than acute pyelonephritis.

There are very few reports in the literature about the use of ${ }^{67}$ Gallium for evaluation of RT patients, and its use has not always been appropriate. Maderazo et al. (1988) observed that the high rate of true negative studies in RT patients was due to early and unwarranted use of ${ }^{67}$ Gallum in patients with fever. Tsang et al. (1989) established the value of ${ }^{67}$ Gallium for detecting and locating the UTI site in native kidneys from RT patients with polycystic renal disease. George et al. (1975) stated that ${ }^{67}$ Gallium could be useful in evaluation of kidney function, graft rejection, and renal inflammatory disease in general.

There is no general agreement about whether an uncomplicated RT should take up ${ }^{67}$ Gallium or not, and to what extent. George et al. (1975) and Frankel et al. (1975) showed no ${ }^{67}$ Gallium deposit more than 24 hours after administration. In a prospective study involving 120 patients with type 1 diabetes and a dual kidney and pancreas transplant, in whom serial biopsies of the renal graft were performed at 2, 6, and 12 months after transplant and yearly thereafter until 10 years, a significant lymphocytic infiltrate, tubulitis, and subclinical rejection were encountered in $60.8 \%$ at one month, in $45.7 \%$ at three months, and in $25.8 \%$ at one year, with a subsequent mean value of $17.7 \%$. Beyond the first year, histological patterns of graft damage changed to reflect microvascular and glomerular damage, and a minimum or absent inflammatory activity was detected after one year. All these histological changes have been called chronic graft nephropathy and consist of two different phases of damage occurring at different times and histological components after the RT. Early tubulointerstitial damage is correlated with immunological factors, including severe acute rejection and persistent subclinical rejection with the addition of ischemia-reperfusion damage. Early damage is characterized by progressive arteriolar hyalinosis, ischemic glomerulosclerosis, and interstitial fibrosis (Nankivell et al., 2003). The existence of these histological changes in RT should therefore be taken into account, and the multiple non-infectious causes responsible for a positive ${ }^{67}$ Gallium uptake, even in the transplanted kidney (Table 1), should be known. Some studies have suggested the value of scintigraphy with ${ }^{111}$ Indium-labeled leukocytes in clinical evaluation of RTs, despite the uncertain effects of immunosuppression and/or uremia on leukocyte function (Frick et al., 1979; Forstrom et al., 1981), while other studies acknowledge their limitations and nonspecificity (Sebrechts et al., 1986).

Sebrechts et al. (1986) conducted a retrospective study on ten patients with RT and fever receiving immunosuppressive treatment for rejection, acute tubular necrosis (ATN), or UTI. They found that all subjects showed a mild, diffuse uptake with ${ }^{111}$ In-labeled leukocytes, and in two of the subjects, focal deposits of greater intensity existed as a result of a segmental infarction and a perinephritic hematoma. Six patients had rejection, two ATN, and four had recently experienced an UTI. Scintigraphy could not distinguish the different conditions. Other studies have shown a diffuse uptake in patients with normally functioning renal grafts (Collier et al., 1984), kidneys from uremic patients, and patients with cyclosporin-induced nephrotoxicity (Fawwaz et al., 1984; McAfee et al., 1985). This uptake, as occurs with ${ }^{67}$ Gallium, appears to represent a non-specific inflammatory response to a variety of immune, ischemic, nephrotoxic, and/or infectious insults of a different severity (Table 2). 
Table 1 - Causes of kidney uptake with ${ }^{67}$ Gallium.

\section{Nature of deposit}

- Primary renal neoplasm

- Metastasis

- Renal abscess

- Pyelonephritis

- Lymphoma/Leukemia

- Drug-induced interstitial nephritis

- Acute interstitial nephritis

- Lupus nephritis

- Sjögren's syndrome

- IgA nephropathy

- Nephrotic syndrome

- Infected polycystic kidney disease

- Glomerulonephritis

- ATN

- Acute rejection

- Chronic rejection

- Heparin therapy

- Obstructive uropathy

- Sarcoidosis

- End-stage renal disease

- Vasculitis focal

focal

focal

diffuse/focal

diffuse and bilateral

bilateral

diffuse

diffuse

bilateral

bilateral

bilateral

focal

diffuse

diffuse

diffuse

diffuse

diffuse and bilateral

diffuse

diffuse and bilateral

diffuse

diffuse and bilateral

Table 2 - Causes of positive uptake in RT with ${ }^{111}$ Indium-labeled leukocytes.

- Normally functioning graft

- ATN

- Cyclosporin A nephrotoxicity

- Acute rejection

- Bacterial or viral infection

- Renal infarction

Lung accumulation of labeled leukocytes is nonspecific (Cook et al., 1984) and appears to have an uncertain diagnostic value in RT patients. Forstrom et al. (1981) found pulmonary uptake in 13 out of 14 patients with CMV infection, but also in 5 out of 8 patients with no evidence of CMV infection. Sebrechts et al. (1986) also found such deposits in patients with no infection or other signs of lung disease. It has been recognized that different systemic conditions such as sepsis, adult respiratory distress syndrome, trauma, pancreatitis, and HD cause a complement-mediated sequestration of leukocytes in pulmonary circulation (Craddock et al., 1977; Rinaldo et al., 1982; Ascher et al., 1979).

Because of such difficulties for interpretation, Sebrechets et al. (1986) found that the only type of focal deposit with ${ }^{111}$ In-labeled leukocytes fully sensitive and specific for active infection in RT patients was surgical wound infection. This deposit is easily differentiated from the mild deposit related to a normal scar (Colleman et al., 1980). In addition, labeled leukocytes, unlike ${ }^{67}$ Gallium, do not accumulate in normal surgical wounds, except for wounds healing by second intention (granulomatous), ostomies, and skin grafts (Palestro et al., 1999). No studies using granulocyte antibodies for assessing these conditions were encountered. 


\section{METHODS FOR IMAGE ACQUISITION AND INTERPRETATION}

\section{Image acquisition}

In HD patients:

- If a pseudoaneurysm is known to exist, perform vascular dynamic scintigraphy before the static study with labeled leukocytes to determine its exact location.

- If vascular activity persists in a 3-hour image, late 6-24 hour images are recommended.

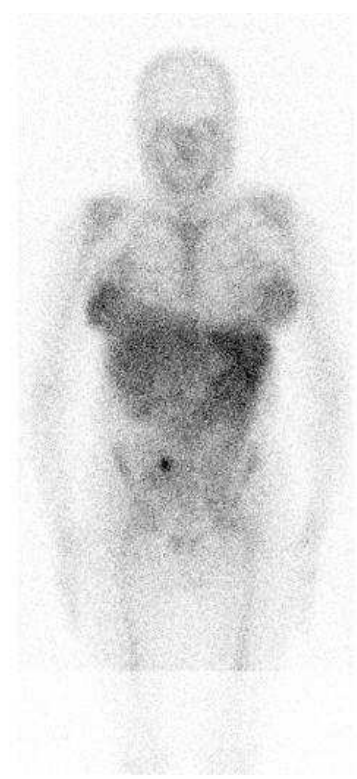

Figure 4 - Positive uptake with ${ }^{67} \mathrm{Ga}$ (planar image). RT patient with cutaneous fistula infection (focal deposit in right iliac fossa).

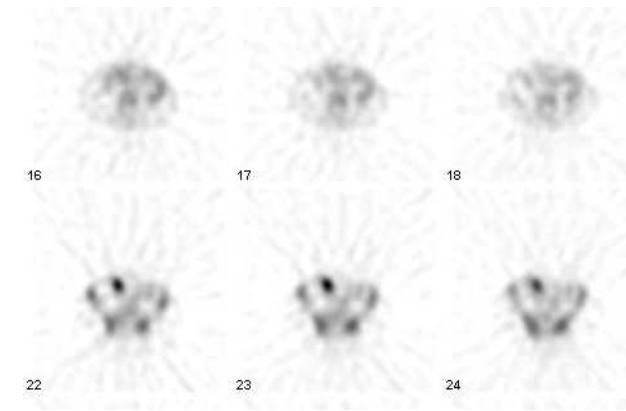

Figure 5 - Transaxial sections of abdominal SPECT study in Fig. 4 (superficially located focal deposit in right iliac fossa)
In CAPD patients:

- If catheter hole/tunnel infection is suspected, obtain static scintigraphic images of abdomen in a lateral view, focused on the catheter hole, as a complement to the previous view, because this allows for measuring depth of the skin deposit.

- When both catheter infection and peritonitis are suspected, liver and spleen must be excluded from the image to minimize potential interference by count stealing.

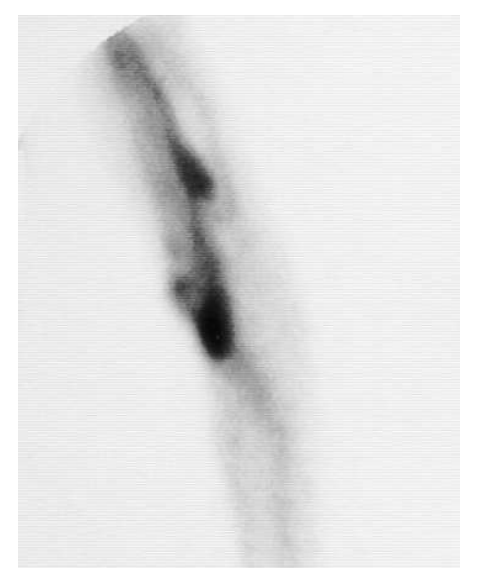

Figure 6 - Infected arteriovenous fistula. Scintigraphy with ${ }^{99 \mathrm{~m}}$ Tc-HMPAO-labeled leukocytes (positive, strong uptake in the entire fistulous tract of the forearm).

Table 3 - Causes of false positives in scintigraphy with labeled leukocytes for assessing vascular prostheses.

- pseudoaneurysm

- intraprosthetic thrombus

- hematoma

- early postoperative period

- superficial prosthesis 
In RT patients:

- Planar image, located in the pelvic region in an anterior view. A tomographic study (SPECT), particularly with SPECT-CT fusion imaging, is sometimes useful to assess an infectious condition in the vicinity of the RT (Figs. 4 and 5).

- Avoid urination in the two hours prior to image acquisition to evaluate excretory activity (George et al., 1976).

No significant differences have been found between images obtained at 48 hours and 72 hours (Hsieh et al., 2001).

\section{Study interpretation}

In HD patients:

Study positive for infection: focal uptake persisting even in 24-hour image, and exclusion of any cause for a false positive result (Table 3 ). (Fig. 6).

\section{In CAPD patients:}

- Study positive for exit hole infection: focal uptake of radiolabeled leukocytes in abdominal wall that coincides in location with the catheter exit hole, and is seen in both the early and late images, with its relative intensity persisting or increasing as compared to the background of late image. (Fig. 7).

- Study positive for catheter tunnel infection: focal uptake at hole level, but more extensive and deeper in character (Fig. 8).

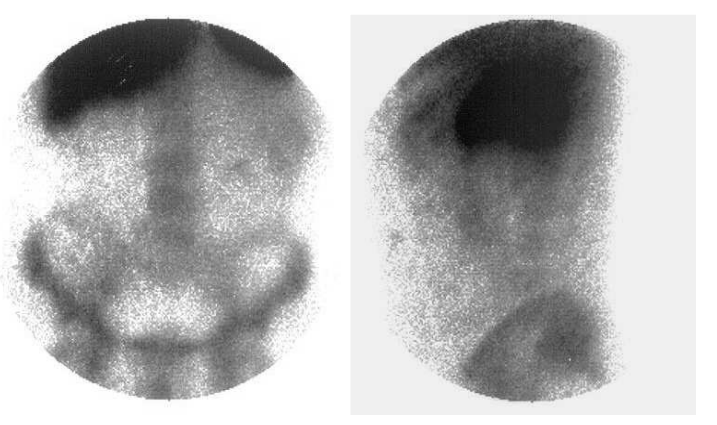

Figure 7 - Exit site infection. Scintigraphy with ${ }^{99 \mathrm{~m}}$ Tc-HMPAO-labeled leukocytes in anterior and lateral views of the abdomen (superficially located focal deposit in region near left flank).
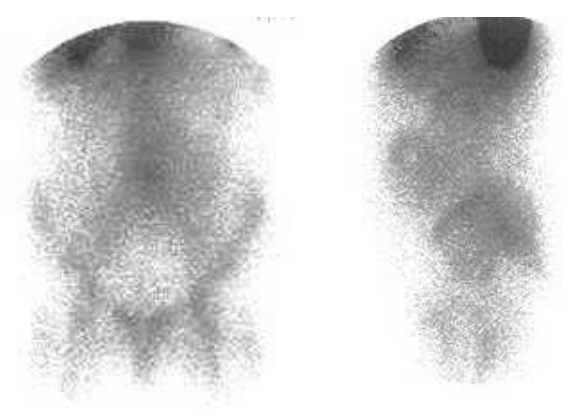

Figure 8 - Tunnel infection. Scintigraphy with ${ }^{99 \mathrm{~m}}$ Tc-HMPAO-labeled leukocytes in anterior and lateral views of the abdomen (extensive, deep uptake in left flank)

- Study positive for peritonitis: diffuse increase in abdominal uptake in both the early and late images (Fig. 9)

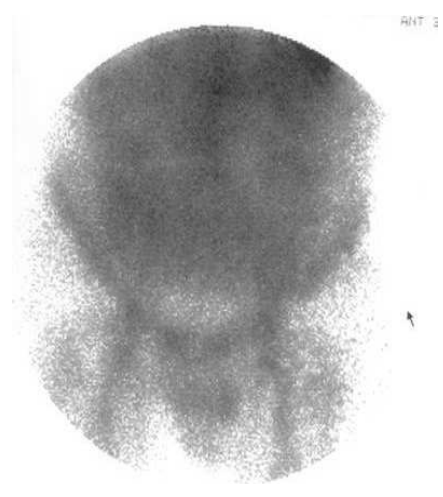

Figure 9. Peritonitis. Scintigraphy with ${ }^{99 \mathrm{~m}} \mathrm{Tc}-$ HMPAO-labeled leukocytes in an anterior view of the abdomen.

In RT patients:

- Positive study: increased uptake with no evidence of associated bladder uptake (Fig. 10).

There are different factors to be considered, because some of them may alter renal biodistribution:

- Concomitant antibiotic therapy may cause false negatives (Schwab et al., 1987; Hilson et al., 1979).

- Presence of neoplasm, exposure to chemotherapy or radiotherapy, or multiple transfusions may increase the chance of 
renal visualization at 24 hours, thus causing false positives (García et al., 1984). An increased and prolonged renal uptake of ${ }^{67}$ Gallium appears to be associated to changes in serum binding protein.

Radiotherapy or chemotherapy decrease serum capacity for ${ }^{67}$ Gallium uptake (Fletcher et al., 1975). In patients with multiple transfusions, partial or total saturation of iron binding protein may explain the high renal uptake (Engelstad et al., 1982). A similar situation may occur in hemochromatosis (Hoffer et al., 1978).

By contrast:

Immunosuppressive therapy, particularly with glucocorticoids, liver failure, or long-term hospitalization do not influence 67 Gallium uptake (Maderazo et al., 1988).

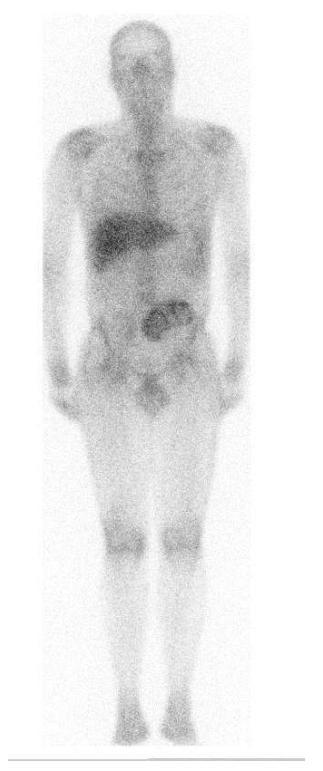

Figure 10 - Glomerulonephritis. Positive uptake by transplanted kidney in left iliac fossa.

The hemodialysis process has no effect on the diagnostic interpretation provided by67Gallium. Marlette et al. (1980) found that HD extracts only $0.5 \%$ of the injected dose by ultrafiltration, much less than the amount eliminated by urinary excretion. Therefore, the radioactive drug may be administered even before the start of HD with no interference with the final result.

\section{CONCLUSION}

Scintigraphy with ${ }^{67}$ Gallium and labeled leukocytes are generally useful in this group of patients as procedures to search for infectious and inflammatory conditions, particularly when localizing signs or symptoms are absent, and may serve to direct attention to given areas in CT or ultrasonography. In patients on HD or CAPD, scintigraphy with labeled leukocytes would be particularly indicated for assessing infectious complications associated to catheters, the main entry portal for pathogens. In RT patients, scintigraphy with ${ }^{67}$ Gallium has limitations for assessing infectious complications in the renal graft because of its low specificity, and is mainly indicated for assessing infectious complications in the surgical wound and perirenal abscesses.

\section{RESUMO}

Pacientes em estágio final de doença renal têm duas opções terapêuticas, diálise e transplante renal. Complicações infecciosas que ocorrem em cada paciente são as principais causas de morbidade e mortalidade nestes casos. Conhecimentos das vantagens e limitações de técnicas nucleares são essenciais para o acompanhamento nestas condições.

\section{REFERENCES}

Abbott, K. C. (2004), Late urinary tract infection after renal transplantation in the United States. Am. J. Kidney Dis., 44, 353-362.

Abbott, K. C. (2001), Hospitalizations for bacterial septicemias after renal transplantation in the United States. Am. J. Nephrol., 21, 122-127.

Ascher, N. L. (1979), Indium ${ }^{-111}$ autologous tagged leukocytes in the diagnosis of intraperitoneal sepsis. Arch. Surg., 114, 386-392.

Ayus, J. C. (1998), Silent infection in clotted hemodialysis access grafts. J. Am. Soc. Nephrol., 9, 1314-1317.

Beathard, G. A. (1999), Management of bacteremia associated with tunneled-cuffed hemodialysis catheters. J. Am. Soc. Nephrol., 10, 1045-1049. 
Becker, W. (1987), Indium ${ }^{-111}$ white blood cell scan in the diagnosis of infectious complications in patiens undergoing regular dialysis treatment. Contributions to Nephrology, ed Bischof-Delaloye, 56, 191-195.

Burgos, F. J. (1999), Transplante renal e infección urinaria. Revisión, Actas Urol. Esp., 23, 95-104.

Brugh, R. (1979), Gallium ${ }^{-67}$ scanning and conservative treatment in acute inflammatory lesions of the renal cortex. J. Urol., 21, 232-235.

Cook, P. S. (1984), Pulmonary uptake in indium ${ }^{-11}$ leukocyte imaging: clinical significance in patients with suspected occult infections. Radiology, 150, 557-561.

Colleman, R. E. (1980), Indium ${ }^{-111}$ labeled leukocytes in the evaluationof suspected abdominal abscesses. Am. J. Surg., 139, 99-104.

Collier, B. D. (1984), Concentration of $\mathrm{In}^{-111-}$ oxine labeled autologous leukocytes in noninfected and nonrejectin renal allografts: concise communication. J. Nucl. Med., 25, 156-159.

Craddock, P. R. (1977), Hemodialysis leukopenia: pulmonary vascular leukostasis resulting from complement activation by dialyzer cellophane membranes. J. Clin. Invest., 59, 879-888.

Engelstad, B. (1982), Altered gallium $^{-67}$ citrate distribution in patients with multiple red blood cell transfusion. Am. J. Rontegenol., 139, 755-759.

Fawwaz, R. A. (1984), Clinical utility of labeled cells for detection of allograft rejection and myocardial infarction. Semin. Nucl. Med., 14, 198-207.

Fishman, J. A. (1998), Infection in organtransplant recipients. N. Engl. J. Med., 338, 1741-1751

Fletcher, J. W. (1975), $\mathrm{Ga}^{-67}$ citrate distribution following whole-body irradiation or chemotherapy. Radiology, 117, 709-712.

Frankel, R. S. (1975), Renal localization of gallium ${ }^{-67}$ citrate. Radiology, 114, 393-397.

Franz, M. (1992), High incidence of significant bacteriuria in kidney graft recipients with triple therapy. Transplant Porc., 24, 289-291.

Frick, M. P. (1979), Use of 11In labeled leukocytes in evaluation of renal transplant rejection: a preliminary report. Clin. Nucl. Med., 4, 24-25.

Forstrom, L. A. (1981), In 11 labeled leukocytes in diagnosis of rejection and cytomegalovirus infection in renal transplant patients. Clin. Nucl. Med., 6, 146-149.

García, J. E. (1984), The spectrum of Gallium ${ }^{-67}$ renal activity in patients with no evidence of renal disease. J. Nucl. Med., 25, 575-580.

George, E. A. (1975). ${ }^{67} \mathrm{Ga}$ citrate in renal allograft rejection. Radiology, 117, 731-733.

George, E. A. (1976), Comparative evaluation of renal transplant rejection with radioiodinated fibrinogen, 99Tc-sulfur colloid and ${ }^{67} \mathrm{Ga}$ citrate. J. Nucl. Med., 17, 175-180.
Gelrud, L. (1974), The kinetics of 67 gallium incorporation into inflammatory lesions, Experimental and clinical studies. J. Lab. Clin. Med., 83, 489-452.

Hilson, A. J. W. (1979), Gallium ${ }^{-67}$ scan in pyrexia of unknown origin. Br. Med. J., 2, 1330-1331.

Hoen, B. (1998), Epibacdial: a multicenter prospective study of risk factors for bacteremia in chronic hemodialysis patiens. J. Am. Soc. Nephrol., 9, 969-876.

Hoffer, P. B. (1978), Gallium ${ }^{-67}$ imaging. New York : Wiley.

Hsieh, J. F. (2001), Effect of imaging time on gallium renal scan. Nucl. Med. Commun., 22, 277-279.

Hurwitz, S. R. (1976), Gallium ${ }^{-67}$ imaging to localize urinary-tract infections. Br. J. Radiol., 49, 156-160.

Kairaitis, L. K. (1999), Outcome and complications of temporary haemodialysis catheters. Nephrol. Dial. Tansplant., 14, 1710-1704.

Kaplowitz, L. G. (1988), A prospective study of infectios in hemodialysis patients: patiens hygiene and other risk factors for infection. Infect. Control. Hosp. Epidemiol., 9, 534-541.

Kessler, M. (1993), Bacteriemia in patiens on chronic hemodialysis: a multicenter prospective survey. Nephron, 64, 95-100.

Keogh, A. M. (1998), Complications in peritoneo dialysis: peritonitis and exit-site infections. Perit. Dial. Int., 16, 464-7.

Kipper, S. L. (1984), $\operatorname{In}^{-111}$ leukocyte scintigraphy for detection of infection associated with peritoneal dialysis catheters. Radiology, 151, 491-4.

Kovalik, E. (1996), A clustering of cases of spinal epidural abscess in hemodialysis patiens. J. Am. Soc. Nephrol., 7, 2264-2267.

Kumar, B. (1976), Significance of delayed $\mathrm{Ga}^{-67}$ localization in the kidneys. J. Nucl. Med., 17, 872-875.

McAfee, J. G. (1985), $\operatorname{In}^{-111}$ labeled leukocytes: a review of problems in image interpretation. Radiology, 155, 221-229.

Maderazo, E. G. (1988), The influence of various factors on the accuracy of Gallium ${ }^{-67}$ imaging for occult infection. J. Nucl. Med., 29, 608-615.

Mailloux, L. (1991), Mortality in dialysis patients: analysis of the causes of death. Am. J. Kidney Dis., 30, 326-335.

Marlette, J. M. (1980), Effect of hemodialysis on Gallium ${ }^{-67-}$ Citrate scanning. Clin. Nucl. Med., 5, 401-403.

Marr, K. A. (1998), Incidence and outcome of Staphylococcus aureus bacteriemia in hemodialysis patiens. Kidney Int., 54, 1684-1689.

Mendez, G. (1980), Gallium ${ }^{-67}$ tomographic radionuclide imaging in acute pyelonephritis. Am. J. Radiol., 134, 17-22.

Meller, J. (2002), Nuclear medicine studies in the dialysis patient. Semin in Dialysis, 15 : (4), 269-276. 
Montenegro, J. (1997), Diálisis peritoneal en la insuficiencia renal crónica. In: Llach, F. And Valderrábano, F. (Eds.). Insuficiencia renal crónica. Diálisis y trasplante renal. Madrid : Ediciones Norma. pp. 999-1047.

Moss, A. H. (1990), Use of a silicone dual-lumen catheter with a dacron cuff as a long-term vascular access for hemodialysis patiens. Am. J. Kidney Dis., 16, 211-215.

Muller, V. (1998), Do urinary tract infections trigger chronic kidney transplant rejection in man? J. Urol., 159, 1826-1829.

Nassar, G. M. (2001), Infectious complications of the hemodiálisis access, Kidney Internacional.,60, 1-13.

Nassar, G. M. (2000), Clotted arteriovenous grafts. A silent source of infection. Semin. Dial., 13,1-3.

Nankivell, B. J. (2003), The natural history of chronic allograft nephropathy. N. Engl. J. Med., 349, 2326-2333.

Neumann, R. D. (1996), Gallium ${ }^{-67}$ imaging for detection of malignant disease. Ed Sander MP, Patoon JA. Baltimore: Williams Wilkins, 1243-1260.

Lawrence, P. R. (1985), Indium ${ }^{-111}$ labeled leukocyte scanning for detection of prosthetic vascular graft infection. J. Vasc. Surg., 2, 165-173.

Lin, W. Y. (2000), Semi-quantitative evaluation of gallium-67 scintigraphy in lupus nephritis. Eur. J. Nucl. Med., 27, 1626-1631.

Linton, A. L. (1980), Acute interstitial nephritis due to drugs. Ann. Intern. Med., 93, 735-741.

Obrador, G. T. (1996), Spinal epidural abscess in hemodialysis patiens: Report of three cases and review of the literature. Am. J. Kidney Dis., 27, 7583.

Palestro, C. J. (1999), Radionuclide imaging of nonosseous infection. Q. Nucl. Med., 43, 46-60.

Palestro, C. J. (1990), Indium-111-labeled leukocyte scintigraphy in hemodialysis access-site infection. J. Nucl. Med., 31, 319-324.

Park, H. (1977), Localization of ${ }^{67} \mathrm{Ga}$ in renal microabscesses. J. Nucl. Med., 18, 313-4.

Piraino, B. (1997), Infectious complications of peritoneal dialysis. Perit. Dial. Int., 17 : (Suppl. 3), 15-8.

Powe, N. R. (1999), Septicemia in dialysis patients: incidence, risk factors and prognosis. Kidney Int., 55, 1081-1090.

Prats, E. (2005), La medicina nuclear en el diagnóstico de la infección de prótesis vascular. In: MartínComín, J. (Ed.). Diagnóstico de la inflamación y de la infección en medicina nuclear. pp. 145-153.

Ramsey, D. (1979), Urinary tract infections in kidney transplant recipients. Arch. Surg., 114, 1022-1025.

Rinaldo, J. E. (1982), Adult respiratory-distress syndrome: changing concepts of lung injury and repair. N. Engl. J. Med., 306, 900-909.

Robinson, D. L. (1997), Bacterial endocarditis in hemodialysis patiens. Am. J. Kidney Dis., 30, 521-524.
Rubin, R. H. (1988), Opportunistic infections in renal allograft recipients. Transplant Proc., 20, 12-18.

Ruiz, S. (2004), Diagnóstico de las complicaciones infecciosas de la diálisis peritoneal contínua ambulatoria mediante leucocitos marcados con ${ }^{99 \mathrm{~m}} \mathrm{Tc}$ HMPAO. Rev. Esp. Med. Nucl., 23, 403-413.

Schwab, S. J. (1999), The hemodialysis catheter conundrum: hate living with them, but can't live without them. Kidney Int., 56, 1-17.

Schwab, S. J. (1987), Diagnosis of cyst infection in polycystic kidney disease. Kidney Int., 31, 216-18.

Sebrechts, C. (1986), Limitations of Indium $^{-111}$ leukocyte scanning in febrile renal transplant patients. AJR., 146, 823-829.

Simon, D. M. (2001), Infectious complications of solid organ transplantations. Infect. Dis. Clin. North. Am., 15, 521-549.

Schmaldienst, S. (2002), Urinary tract infections after renal transplantation. Cuur. Opin. Urol., 12, 125-130.

Torregrosa J. V. (1994), The usefulness of ${ }^{111-}$ In-labeled platelet scintigraphy in the diagnosis of patients with febrile syndrome and a nonfunctioning renal graft. Transplantation, 57, 1732-1735.

Tsan, M. (1978). Studies on the gallium accumulation in inflammatory lesions: gallium uptake by human polymorphonuclear leukocytes, 19, 36-39.

Tsang, V. (1989), Gallium ${ }^{-67}$ scintigraphy in the detection of infected polycystic kidneys in renal transplant recipients. Nucl. Med. Commun., 10, 167-170.

Twardowski, Z. J. (1993), Peritoneal catheter exit-site and tunnel infections. In: Nissenson, A. R. and Fine, R. N. (Eds.). Dialysis therapy. $2^{\text {nd }}$ ed. Philadelphia : Hanley \& Belfus. pp. 165-168.

Vargemezis, V. (2001), Prevention and management of peritonitis and exit-site infection in patients on continuous ambulatory peritoneal dialysis. Nephrol. Dial. Transplant., 16 : (Suppl 6), 106-8.

Wood, B. J. (1978), Gallium citrate $\mathrm{Ga}^{-67}$ imaging in noninfectious intersticial nephritis. Arch. Intern. Med., 138, 1665-1666.

Wyner, L. M. (1994), The evaluation and management of urinary tract infections in recipients of solid-organ transplants. Seminars in Urology, 12, 1324-139.

Yen, T. C. (1999), The value of $\mathrm{Ga}^{-67}$ renal SPECT in diagnosing and monitoring complete and incomplete treatment in children with acute pyelonephritis. Clin. Nucl. Med., 24, 669-673. 